\title{
Terahertz Sum-Frequency Excitation of a Raman-Active Phonon
}

\author{
Sebastian Maehrlein, ${ }^{1, *}$ Alexander Paarmann, ${ }^{1}$ Martin Wolf, ${ }^{1}$ and Tobias Kampfrath ${ }^{1,2}$ \\ ${ }^{1}$ Department of Physical Chemistry, Fritz. Haber Institute of the Max Planck Society, Faradayweg 4-6, 14195 Berlin, Germany \\ ${ }^{2}$ Department of Physics, Freie Universität Berlin, Arnimallee 14, 14195 Berlin, Germany \\ (Received 9 March 2017; revised manuscript received 25 June 2017; published 22 September 2017)
}

\begin{abstract}
In stimulated Raman scattering, two incident optical waves induce a force oscillating at the difference of the two light frequencies. This process has enabled important applications such as the excitation and coherent control of phonons and magnons by femtosecond laser pulses. Here, we experimentally and theoretically demonstrate the so far neglected up-conversion counterpart of this process: $\mathrm{THz}$ sumfrequency excitation of a Raman-active phonon mode, which is tantamount to two-photon absorption by an optical transition between two adjacent vibrational levels. Coherent control of an optical lattice vibration of diamond is achieved by an intense terahertz pulse whose spectrum is centered at half the phonon frequency of $40 \mathrm{THz}$. Remarkably, the carrier-envelope phase of the THz pulse is directly transferred into the phase of the lattice vibration. New prospects in general infrared spectroscopy, action spectroscopy, and lattice trajectory control in the electronic ground state emerge.
\end{abstract}

DOI: 10.1103/PhysRevLett.119.127402

Gaining control over ultrafast elementary motions of electrons, spins, and ions in solids is of major scientific and applied interest. Examples include steering of chemical reactions along a desired pathway [1] and switching of spins in magnetically ordered materials [2,3]. Many control strategies take advantage of terahertz $(\mathrm{THz})$ modes of the material. An ubiquitous example is lattice vibrations (phonons) whose control by femtosecond laser pulses $[4,5]$ has enabled new pathways to permanent material modification [6], insulator-to-metal transitions [7,8], and magnetization manipulation [2,9].

For selective excitation of coherent lattice vibrations, schemes based on one- or two-photon interactions are established [see Figs. 1(a) and 1(b)]. In the most straightforward approach, the laser electric field resonantly drives the electric dipoles of a long-wavelength lattice mode. This onephoton absorption (1PA) process requires an infrared-active transition and a pump spectrum that covers the phonon frequency $\Omega / 2 \pi$, typically between $\sim 1$ and $40 \mathrm{THz}$ [Fig. 1(a)] [10-13]. To date, most works have implemented lattice excitation using stimulated Raman scattering (SRS) of a light pulse containing frequencies $\omega_{1}^{\prime}$ and $\omega_{2}^{\prime}=\omega_{1}^{\prime}+\Omega$, both at roughly $2 \pi \times 500 \mathrm{THz}$ [Fig. 1(b)]. The two rapidly oscillating light-field components couple primarily to electrons of the material [14], either nonresonantly (in optically transparent solids) $[15,16]$ or resonantly (in opaque solids) $[17,18]$, resulting in a force that coherently drives the lattice at the difference frequency $\omega_{2}^{\prime}-\omega_{1}^{\prime}=\Omega$. Such rectification can also be achieved through anharmonic interaction with resonantly driven infrared-active phonons at $\omega_{1}^{\prime}$ and $\omega_{2}^{\prime}$ [19].

Note that the difference-frequency process underlying SRS [Fig. 1(b)] is a familiar phenomenon in nonlinear optics where it is accompanied by the generation of sum-frequency signals [20]. This analogy suggests a novel scheme of coherent lattice control: the two incident frequencies are chosen such that their sum is resonant with the target mode [Fig. 1(c)]. Such sum-frequency excitation (SFE) by a single light pulse is tantamount to two-photon absorption (2PA) and offers similarly attractive features as 1PA. So far, 2PA has been widely used to induce [21,22] and coherently control $[23,24]$ electronic transitions by $\sim 1 \mathrm{eV}$ photons. However, 2PA by vibrational resonances such as optical phonons has not yet been demonstrated because the laser frequencies used ( $\sim 500 \mathrm{THz}$ ) were typically one order of magnitude larger than the highest phonon frequencies.

In this work, we provide a first experimental and theoretical demonstration of SFE of lattice vibrations. An intense $\mathrm{THz}$ pulse with a center frequency of $20 \mathrm{THz}$ is found to coherently drive the long-wavelength Raman-active optical phonon of diamond at the sum frequency of $40 \mathrm{THz}$. Remarkably and similar to 1PA, the carrier-envelope phase (CEP) of the $\mathrm{THz}$ pulse is imprinted into the phase of the lattice vibration. Because of the low $\mathrm{THz}$ photon energy, parasitic electronic excitations are suppressed, thereby opening the way to coherent control of Raman-active modes such as phonons and magnons in the electronic ground state.

Experiment.-In our pump-probe scheme [Fig. 1(d)], a $\mathrm{THz}$ pump pulse with tunable center frequency [25] is directed onto the sample to drive coherent lattice vibrations. The instantaneous time-dependent phonon amplitude is monitored by a time-delayed optical probe pulse measuring the transient birefringence of the material [4,5].

As a sample, we choose a high-purity (100)-oriented diamond single crystal (type IIa, thickness $200 \mu \mathrm{m}$ ) grown by chemical vapor deposition. We focus on the zone-center $F_{2 g}$ optical phonon at $\Omega / 2 \pi \approx 40 \mathrm{THz}$, which is one of the highest phonon frequencies known. This mode has a vanishing electric dipole moment but is strongly Raman 
(a)

(b)

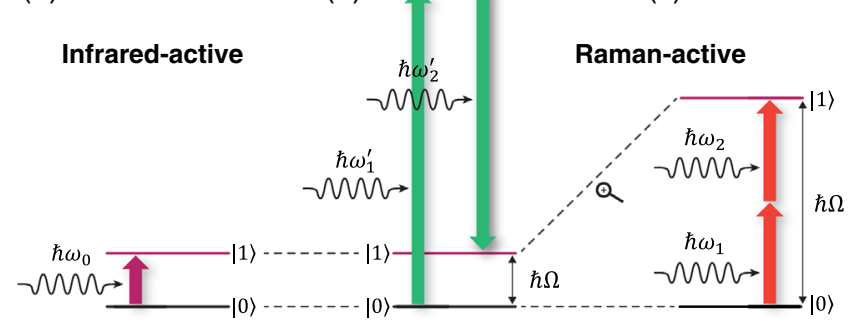

(d)

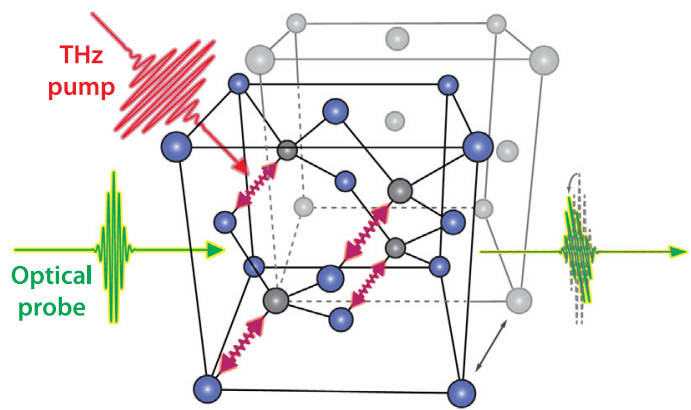

(e)

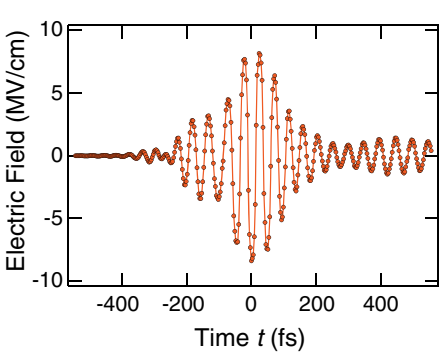

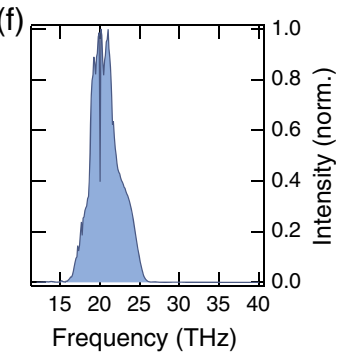

Frequency $(\mathrm{THz})$
FIG. 1. (a)-(c) Schemes for driving coherent phonons. (a) Direct excitation by one-photon absorption (1PA): the pump frequency is resonant with the infrared-active phonon mode. (b) Stimulated Raman scattering (SRS): the difference frequency of two spectral components of the pump field is resonant with a Raman-active phonon mode. (c) Sum-frequency excitation (SFE): the sum frequency of two incident $\mathrm{THz}$ electric-field components is resonant with a Raman-active phonon mode. This process can be considered as two-photon absorption (2PA) by an optical transition between two adjacent vibrational levels. (d) Unit cell of diamond and schematic of the experiment: an intense $\mathrm{THz}$ pulse (red) excites the $F_{2 g}$ mode whose dynamics are monitored by a delayed ultrashort optical probe pulse (green). Red arrows indicate coherent lattice motion corresponding to the Ramanactive, infrared-forbidden $F_{2 g}$ mode at $40 \mathrm{THz}$. (e) Transient electric field and (f) intensity spectrum of a typical $\mathrm{THz}$ pump pulse used in the experiments.

active [26-28]. The underlying symmetric C-C stretch vibrations [Fig. 1(d)] are found in many other $\mathrm{C}-\mathrm{C}$ covalent-bond systems and were subject to several impulsive SRS studies, for instance, on carbon nanotubes [29], graphene [30], and organic molecules [31].

To drive and observe a $40 \mathrm{THz}$ phonon, intense phaselocked $\mathrm{THz}$ pump pulses and very short probe pulses are required. The pump pulse is generated by differencefrequency mixing of two phase-correlated infrared pulses (energy $1.2 \mathrm{~mJ}$, repetition rate $1 \mathrm{kHz}$ ) from two optical parametric amplifiers in a GaSe crystal [25]. A typical pump pulse and its intensity spectrum are shown in Figs. 1(e) and 1(f), respectively. The CEP of the inherently phase-locked pulse can be set by the delay between the two generating infrared pulses [25]. The pump-induced coherent-phonon dynamics are sampled by a synchronized ultrashort probe pulse (duration $7 \mathrm{fs}$, center wavelength $750 \mathrm{~nm}$ ) that measures the transient birefringence of the sample [32]. Pump and probe traverse the sample collinearly under normal incidence. Their linear polarizations are set to an angle of $45^{\circ}$ with respect to each other. To obtain the pump electric field of Fig. 1(e), the diamond sample is replaced by a GaSe crystal $[25,33,34]$. All experiments are performed at room temperature and under ambient conditions.

Coherent-phonon signals.-Figure 2 shows transient optical birefringence signals $S(t)$ obtained for three different center frequencies $\omega_{0}$ of the pump pulse: $\Omega, 0.75 \Omega$, and $0.5 \Omega$. When the pump center frequency is resonant with the target mode [Fig. 2(a)], we observe a peak around $t=0$, whose shape approximately follows the intensity envelope of the pump pulse [Fig. 2(b)]. It arises from the instantaneous nonresonant response of the diamond electrons to the $\mathrm{THz}$ pump pulse. Apart from this Kerr effect [35], however, we do not observe any features indicative of a coherent lattice vibration. When we lower the pump center frequency from $\omega_{0}=\Omega$ to $0.75 \Omega$ [Fig. 2(c)], very similar dynamics are found [Fig. 2(d)].

A strikingly different response is obtained when the pump spectrum is centered at half the phonon frequency [Fig. 2(e)]. For delays $t$ larger than the pump-pulse duration, the transient birefringence is dominated by an oscillatory component [Fig. 2(f)] whose Fourier spectrum exhibits a sharp peak at $40 \mathrm{THz}$ [Fig. 2(e)]. By fitting an exponentially damped harmonic oscillation to $S(t)$ for times $t>200 \mathrm{fs}$, we obtain a center frequency of $\Omega / 2 \pi=39.95 \mathrm{THz}$ and damping constant of $\Gamma=0.283 \mathrm{ps}^{-1}$ (see Fig. S1 of the Supplemental Material [36]). These values agree excellently with those inferred from SRS excitation using optical pump pulses with a center frequency of $750 \mathrm{THz}$ [26], as well as from spontaneous Raman scattering [28] (also see Table S1 [36]). Therefore, the oscillatory pump-probe signal of Fig. 2(f) is a clear hallmark of diamond's $F_{2 g}$ phonon. Its decay is known to result from anharmonic interactions with other lattice modes $[28,44]$.

As seen in Fig. 3(a), the oscillation amplitude is found to grow linearly with the pump power. Therefore, the coherent lattice vibration is the result of two interactions with the THz pump field [20] and can only arise from a difference-frequency process [SRS, Fig. 1(b)] or a sumfrequency process [SFE, Fig. 1(c)]. Since the coherent phonon is only observed for a pump center frequency of $\Omega / 2$, we have provided evidence that we here observe a coherent lattice vibration driven by SFE of a $\mathrm{THz}$ pulse. Importantly, the transient signals do not exhibit any indications of unwanted sample heating in the form of a 

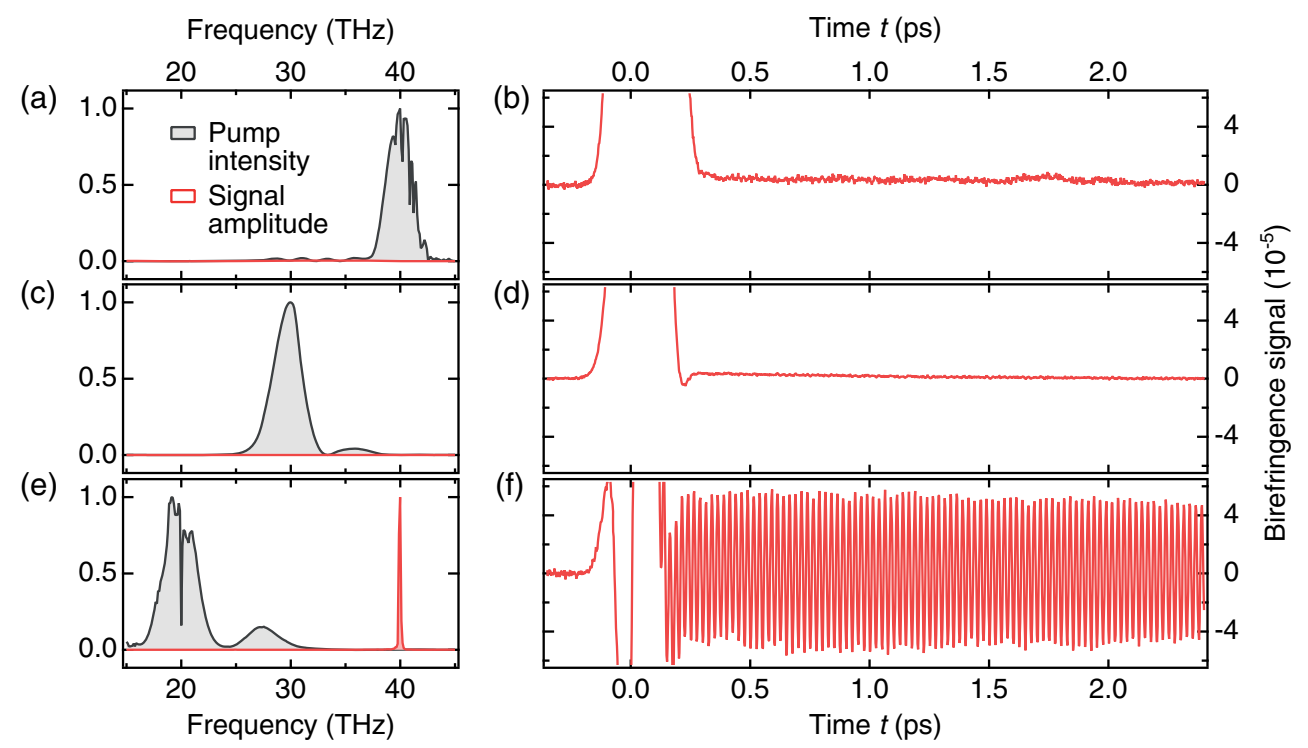

FIG. 2. Sum-frequency excitation (SFE) of a coherent phonon. (a) Spectrum of a pump pulse centered at the phonon frequency $\Omega / 2 \pi \approx 40 \mathrm{THz}$. (b) Resulting pump-probe signal, showing no signature of lattice vibrations. The peak around $t=0$ is due to the instantaneous electronic response to the pump field. Panels (c),(d) and (e),(f) are as with (a),(b) but for a pump center frequency of 0.5 and $0.75 \Omega$, respectively. In panel (f), the long-lived oscillation reveals a coherent phonon at frequency $\Omega / 2 \pi \approx 40 \mathrm{THz}$ as seen in the signal spectrum of panel (e).

slowly varying background, as is often observed with optical excitation $[4,5,15]$.

Model.-To put our interpretation on a theoretical basis, we assume that the solid has only one uniform vibrational mode, an optical phonon characterized by the normal coordinate $Q$ and frequency $\Omega$. The electric field $E(t)$ of the incident pump pulse exerts Coulomb forces on electrons and ions, resulting in a time-dependent electricdipole density (polarization) $P(t)$. Since diamond is an insulator (electronic band gap $5.5 \mathrm{eV}$ ), the material remains in its electronic ground state whose electron density follows the pump field (photon energy $\sim 80 \mathrm{meV}$ ) adiabatically. Consequently, the polarization is fully determined by the instantaneous values of $Q$ and $E$, and we have $P(t)=P(Q(t), E(t))$. We assume a Lagrangian [16] that captures the mode' s kinetic energy $\left(M \dot{Q}^{2} / 2\right)$, potential energy $\left(M \Omega^{2} Q^{2} / 2\right)$, and interaction $\left(V_{c} P E\right)$ with the pump field, where $M$ is the effective mass of the lattice mode and $V_{c}$ is the volume of diamond's unit cell. Linearizing $P$ with respect to $E$ and using the Lagrange equation yields the classical harmonic-oscillator-type equation of motion for $Q$,

$$
\frac{M}{V_{c}}\left(\frac{\partial^{2}}{\partial t^{2}}+2 \Gamma \frac{\partial}{\partial t}+\Omega^{2}\right) Q=E \frac{\partial P}{\partial Q}+E^{2} \frac{\partial \chi}{\partial Q}
$$

which is also obtained by a quantum-mechanical treatment [15]. The right-hand side of Eq. (1) is the sum of two driving forces fully determined by the instantaneous value of $E(t)$. The first term describes the direct coupling of $E$ to the polarization arising from lattice displacement. It corresponds to the 1PA scheme of Fig. 1(a), but vanishes here because the effective charge $V_{c} \partial P / \partial Q$ of the infraredinactive $F_{2 g}$ mode of diamond is zero. The second term results from the coupling of $E$ to the $Q$-dependent part of the field-induced electronic polarization $\chi E$, where $\chi=$ $\partial P / \partial E$ is the linear dielectric susceptibility. Clearly, this coupling is Raman-type because it is mediated by the Raman tensor $\partial \chi / \partial Q$, which is nonzero for the relevant mode $[26,28]$.

Note that the second force term on the right-hand side of Eq. (1) follows the squared pump field $E^{2}(t)$. Therefore, it consists of a superposition of difference- and sumfrequency pairs of the spectrum of $E(t)$. The differencefrequency component, corresponding to conventional SRS [Fig. 1(b)], follows the pump intensity envelope. It has been employed for impulsive excitation of phonons $[4,5,17]$ and magnons [3] in numerous works. As we show here, also the sum-frequency component is capable of driving such Raman-active modes [Fig. 1(c)]. It is precisely this term that describes phonon excitation by THz SFE.

As detailed in the Supplemental Material [36], we solve Eq. (1) for $Q(t)$ and model the transient optical birefringence as a weighted sum of $Q(t)$ and $E^{2}(t)$, the latter capturing the instantaneous electronic response (THz Kerr effect [35]). A final temporal convolution with a square function accounts for the velocity mismatch of pump and probe pulses propagating through the sample [32]. Using this procedure and the actual driving field of Fig. 1(e), we obtain the gray line of Fig. 3(b). The agreement with the measured signal (blue line) is striking, in particular during temporal overlap of pump and probe pulses. Our model confirms that the sharp dip in the pump spectrum [Figs. 1(f) 

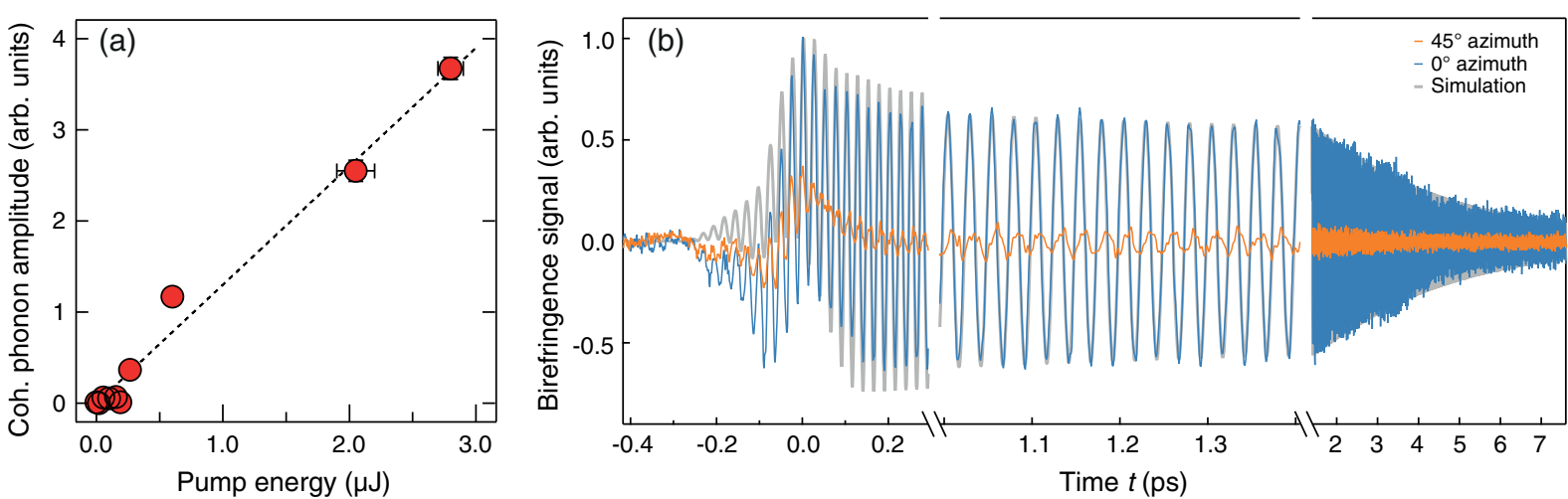

FIG. 3. (a) Pump-energy dependence of the coherent phonon amplitude. (b) Measured (blue line) and simulated (gray line) THz-pulseinduced phonon dynamics [see Eq. (1) and text for model details]. Azimuthal sample rotation by $45^{\circ}$ leads to an almost vanishing phonon signal (orange curve), consistent with the Raman-tensor symmetry of diamond [26]. The instantaneous electronic response $(t \approx 0)$ is less prominent than in Fig. 2 since an optimized pump spectrum [Fig. 1(e)] was used (also see Supplemental Material [36]).

and 2(e)], which arises from absorption by $\mathrm{CO}_{2}$ in ambient air, has negligible impact on the phonon dynamics [36].

The scaling of the pump-probe signal with the Raman tensor [see Eq. (1)] is consistent with its dependence on sample orientation $[26,28]$. For example, when the sample is rotated from the azimuth of largest phonon signal amplitude [blue line in Fig. 3(b)] by an angle of $45^{\circ}$, we find almost complete suppression of the oscillatory component [orange line in Fig. 3(b)], in agreement with the symmetry of the (100)-oriented diamond crystal [26]. Therefore, our results fully support the notion that we have observed THz SFE as a new mechanism for launching of a coherent phonon.

Coherent-phonon phase control.-Our experiment allows us to explore the impact of the CEP on the SFE process, which is expected to be very different from the SRS case. A CEP change by $\Delta \varphi_{0}$ shifts the phase of the phonon oscillation by $\Delta \varphi_{0}-\Delta \varphi_{0}=0$ in the differencefrequency-type case [SRS, Fig. 1(b)], but by $\Delta \varphi_{0}+\Delta \varphi_{0}=$ $2 \Delta \varphi_{0}$ in the sum-frequency process [SFE, Fig. 1(c)]. Unlike SRS, THz SFE should, therefore, enable direct phase control of coherent phonon motion by tuning the CEP of the pump pulse.

To put this expectation to test, we measure phonon dynamics as a function of the CEP as shown in Fig. 4. For example, when the CEP changes by $\Delta \varphi_{0}=\pi / 2$ [Fig. 4(a), green line], the phonon oscillation is found to undergo a phase shift of $\pi$ [Fig. 4(b), green line]. The data of Figs. 4 and S2 of the Supplemental Material [36] demonstrate that twice the CEP shift of the pump field is directly transferred into the resulting lattice motion. These results and Eq. (1) highlight an interesting similarity to 1PA-driven dynamics, which are also sensitive to the pump CEP. Another common feature of the 1PA [Fig. 1(a)] and 2PA [Fig. 1(c)] scheme is that they permit nonimpulsive excitation with a pump pulse much longer than one phonon oscillation cycle.

Discussion.-Neither 2PA nor SFE have so far been observed for an optical transition between adjacent vibrational levels. Here, we witness the full dynamics of this process, starting with the coherent SFE of the vibration, the subsequent decoherence and absorption. In contrast to previous quantum-coherent studies of $2 \mathrm{PA}$ by electronic transitions $[23,24,45]$, the excited coherent-phonon state of this work has a much lower frequency and decoherence rate. Therefore, its dynamics can be sampled by a femtosecond optical probe directly in the time domain.

In terms of applications, the selective energy deposition in Raman-active modes by $\mathrm{THz} 2 \mathrm{PA}$ may enable machining of transparent materials by midinfrared industrial lasers. We estimate [36] that the 2PA strength by the diamond

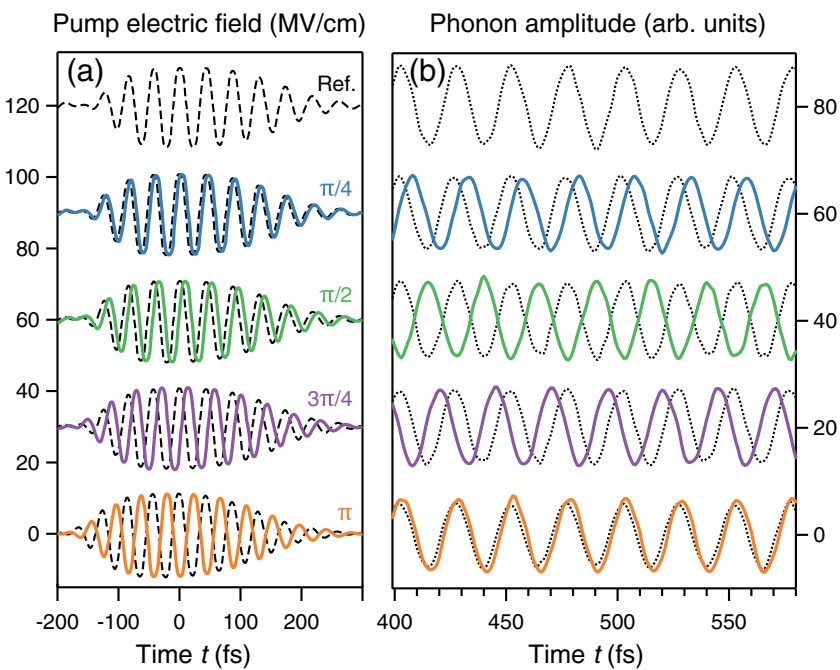

FIG. 4. Demonstration of phonon phase control by tuning the CEP of the THz pump pulse. (a) Measured pump electric field for five CEP settings from 0 to $\pi$ (solid lines). For comparison, the $\Delta \varphi_{0}=0$ reference transient (broken lines) is measured simultaneously for each CEP. (b) Resulting coherent phonon signals (solid lines) measured after sample excitation with the pump fields of panel (a) including reference phonon signals (broken lines). 
phonon is only 1 order of magnitude smaller than that of strong optical two-photon absorbers such as GaAs or CdTe [46]. Moreover, THz SFE will potentially extend established, highly sensitive infrared vibrational spectroscopies to infrared-forbidden yet Raman-active modes. A prominent example is action spectroscopy [47], which relies on detecting modifications the absorbed infrared radiation has induced in the sample.

Remarkably, due to the low photon energies required, $\mathrm{THz}$ SFE provides a unique tool to steer chemical reactions [1] or phase transitions $[7,8]$ in the electronic ground state, solely driven by Raman-active lattice vibrations. Shaping of $\mathrm{THz}$ pulses [48] to multicolor sequences could enable independent phase and amplitude control of the lattice trajectory along multiple normal coordinates, which is challenging with optical pulses accompanied by parasitic electronic excitations [49].

In conclusion, we have demonstrated that coherent lattice motion can be launched and controlled by THz SFE. This approach provides coherent control of all Raman-active modes that have so far been inaccessible by $\mathrm{THz}$ or midinfrared radiation. Simultaneously, parasitic electronic excitation, which occurs when optical laser pulses are used, is greatly reduced. Finally, this so far neglected type of lightmatter coupling extends infrared vibrational spectroscopy methods to infrared-forbidden modes.

We thank Kunie Ishioka for providing us with the diamond sample and the European Union for funding through the ERC-H2020 Grant TERAMAG/No. 681917.

*maehrlein@fhi-berlin.mpg.de

[1] J. L. LaRue, T. Katayama, A. Lindenberg, A. S. Fisher, H. Öström, A. Nilsson, and H. Ogasawara, THz-Pulse-Induced Selective Catalytic CO Oxidation on Ru, Phys. Rev. Lett. 115, 036103 (2015).

[2] T. Kubacka et al., Large-amplitude spin dynamics driven by a $\mathrm{THz}$ pulse in resonance with an electromagnon, Science 343, 1333 (2014).

[3] A. V. Kimel, A. Kirilyuk, P. A. Usachev, R. V. Pisarev, A. M. Balbashov, and T. Rasing, Ultrafast non-thermal control of magnetization by instantaneous photomagnetic pulses, Nature (London) 435, 655 (2005).

[4] T. K. Cheng, S. D. Brorson, A. S. Kazeroonian, J. S. Moodera, G. Dresselhaus, M. S. Dresselhaus, and E. P. Ippen, Impulsive excitation of coherent phonons observed in reflection in bismuth and antimony, Appl. Phys. Lett. 57, 1004 (1990).

[5] G. C. Cho, W. Kütt, and H. Kurz, Subpicosecond timeresolved coherent-phonon oscillations in GaAs, Phys. Rev. Lett. 65, 764 (1990).

[6] C. Klieber, E. Peronne, K. Katayama, J. Choi, M. Yamaguchi, T. Pezeril, and K. A. Nelson, Narrow-band acoustic attenuation measurements in vitreous silica at frequencies between 20 and $400 \mathrm{GHz}$, Appl. Phys. Lett. 98, 211908 (2011).

[7] K. W. Kim, A. Pashkin, H. Schäfer, M. Beyer, M. Porer, T. Wolf, C. Bernhard, J. Demsar, R. Huber, and A. Leitenstorfer, Ultrafast transient generation of spin-density-wave order in the normal state of $\mathrm{BaFe}_{2} \mathrm{As}_{2}$ driven by coherent lattice vibrations, Nat. Mater. 11, 497 (2012).

[8] M. Rini, R. Tobey, N. Dean, J. Itatani, Y. Tomioka, Y. Tokura, R. W. Schoenlein, and A. Cavalleri, Control of the electronic phase of a manganite by mode-selective vibrational excitation, Nature (London) 449, 72 (2007).

[9] J.-W. Kim, M. Vomir, and J.-Y. Bigot, Ultrafast Magnetoacoustics in Nickel Films, Phys. Rev. Lett. 109, 166601 (2012).

[10] T. Huber, M. Ranke, A. Ferrer, L. Huber, and S. L. Johnson, Coherent phonon spectroscopy of non-fully symmetric modes using resonant terahertz excitation, Appl. Phys. Lett. 107, 091107 (2015).

[11] I. Katayama, H. Aoki, J. Takeda, H. Shimosato, M. Ashida, R. Kinjo, I. Kawayama, M. Tonouchi, M. Nagai, and K. Tanaka, Ferroelectric Soft Mode in a $\mathrm{SrTiO}_{3}$ Thin Film Impulsively Driven to the Anharmonic Regime Using Intense Picosecond Terahertz Pulses, Phys. Rev. Lett. 108, 097401 (2012).

[12] T. Qi, Y.-H. Shin, K.-L. Yeh, K. A. Nelson, and A. M. Rappe, Collective Coherent Control: Synchronization of Polarization in Ferroelectric $\mathrm{PbTiO}_{3}$ by Shaped $\mathrm{THz}$ Fields, Phys. Rev. Lett. 102, 247603 (2009).

[13] M. Jewariya, M. Nagai, and K. Tanaka, Ladder Climbing on the Anharmonic Intermolecular Potential in an Amino Acid Microcrystal via an Intense Monocycle Terahertz Pulse, Phys. Rev. Lett. 105, 203003 (2010).

[14] R. Merlin, Generating coherent $\mathrm{THz}$ phonons with light pulses, Solid State Commun. 102, 207 (1997).

[15] L. Dhar, J. A. Rogers, and K. A. Nelson, Time-resolved vibrational spectroscopy in the impulsive limit, Chem. Rev. 94, 157 (1994).

[16] Y. R. Shen and N. Bloembergen, Theory of stimulated brillouin and raman scattering, Phys. Rev. 137, A1787 (1965).

[17] E. M. Bothschafter, A. Paarmann, E. S. Zijlstra, N. Karpowicz, M. E. Garcia, R. Kienberger, and R. Ernstorfer, Ultrafast Evolution of the Excited-State Potential Energy Surface of $\mathrm{TiO}_{2}$ Single Crystals Induced by Carrier Cooling, Phys. Rev. Lett. 110, 067402 (2013).

[18] T. Dekorsy, G. C. Cho, and H. Kurz, in Topics in Applied Physics, edited by M. Cardona and G. Güntherodt (Springer, Berlin, Heidelberg, 2000), Vol. 76, p. 169.

[19] M. Foerst, C. Manzoni, S. Kaiser, Y. Tomioka, Y. Tokura, R. Merlin, and A. Cavalleri, Nonlinear phononics as an ultrafast route to lattice control, Nat. Phys. 7, 854 (2011).

[20] S. Mukamel, Principles of Nonlinear Optical Spectroscopy, Oxford Series in Optical and Imaging Sciences, Vol. 6 (Oxford University Press, New York, 1999), Vol. 6.

[21] A. F. Gibson, C. B. Hatch, P. N. D. Maggs, D. R. Tilley, and A. C. Walker, Two-photon absorption in indium antimonide and germanium, J. Phys. C 9, 3259 (1976).

[22] D. Seo, J. M. Gregory, L. C. Feldman, N. H. Tolk, and P. I. Cohen, Multiphoton absorption in germanium using pulsed infrared free-electron laser radiation, Phys. Rev. B 83, 195203 (2011).

[23] D. Meshulach and Y. Silberberg, Coherent quantum control of two-photon transitions by a femtosecond laser pulse, Nature (London) 396, 239 (1998). 
[24] Y. Silberberg, Quantum coherent control for nonlinear spectroscopy and microscopy, Annu. Rev. Phys. Chem. 60, 277 (2009).

[25] A. Sell, A. Leitenstorfer, and R. Huber, Phase-locked generation and field-resolved detection of widely tunable terahertz pulses with amplitudes exceeding $100 \mathrm{MV} / \mathrm{cm}$, Opt. Lett. 33, 2767 (2008).

[26] K. Ishioka, M. Hase, M. Kitajima, and H. Petek, Coherent optical phonons in diamond, Appl. Phys. Lett. 89, 231916 (2006).

[27] C. V. Raman and K. S. Krishnan, A new type of secondary radiation, Nature (London) 121, 501 (1928).

[28] S. A. Solin and A. K. Ramdas, Raman spectrum of diamond, Phys. Rev. B 1, 1687 (1970).

[29] A. Gambetta, C. Manzoni, E. Menna, M. Meneghetti, G. Cerullo, G. Lanzani, S. Tretiak, A. Piryatinski, A. Saxena, R. L. Martin, and A. R. Bishop, Real-time observation of nonlinear coherent phonon dynamics in singlewalled carbon nanotubes, Nat. Phys. 2, 515 (2006).

[30] K. Ishioka, M. Hase, M. Kitajima, L. Wirtz, A. Rubio, and H. Petek, Ultrafast electron-phonon decoupling in graphite, Phys. Rev. B 77, 121402 (2008).

[31] J.-Y. Bigot, T.-A. Pham, and T. Barisien, Femtosecond time resolved molecular dynamics of a polydiacetylene backbone, Chem. Phys. Lett. 259, 469 (1996).

[32] M. Sajadi, M. Wolf, and T. Kampfrath, Terahertz-fieldinduced optical birefringence in common window and substrate materials, Opt. Express 23, 28985 (2015).

[33] A. Leitenstorfer, S. Hunsche, J. Shah, M. C. Nuss, and W. H. Knox, Detectors and sources for ultrabroadband electrooptic sampling: Experiment and theory, Appl. Phys. Lett. 74, 1516 (1999).

[34] Q. Wu, M. Litz, and X.-C. Zhang, Broadband detection capability of ZnTe electro-optic field detectors, Appl. Phys. Lett. 68, 2924 (1996).

[35] M. C. Hoffmann, N. C. Brandt, H. Y. Hwang, K.-L. Yeh, and K. A. Nelson, Terahertz Kerr effect, Appl. Phys. Lett. 95, 231105 (2009).

[36] See Supplemental Material at http://link.aps.org/ supplemental/10.1103/PhysRevLett.119.127402 for model details and additional data, which includes Refs. [37-43].
[37] A. Schneider, I. Biaggio, and P. Günter, Terahertz-induced lensing and its use for the detection of terahertz pulses in a birefringent crystal, Appl. Phys. Lett. 84, 2229 (2004).

[38] P.E. Martin and E.F. Barker, The infrared absorption spectrum of carbon dioxide, Phys. Rev. 41, 291 (1932).

[39] W. B. Person and G. Zerbi, Vibrational Intensities in Infrared and Raman Spectroscopy (Elsevier Science Ltd., New York, 1982), Vol. 20.

[40] C. Kuebler, R. Huber, S. Tübel, and A. Leitenstorfer, Ultrabroadband detection of multi-terahertz field transients with GaSe electro-optic sensors: Approaching the near infrared, Appl. Phys. Lett. 85, 3360 (2004).

[41] T. Kampfrath, J. Notzold, and M. Wolf, Sampling of broadband terahertz pulses with thick electro-optic crystals, Appl. Phys. Lett. 90, 231113 (2007).

[42] R. W. Boyd, Nonlinear Optics, 3rd ed. (Academic, New York, 2008).

[43] M. D. Levenson, C. Flytzanis, and N. Bloembergen, Interference of resonant and nonresonant three-wave mixing in diamond, Phys. Rev. B 6, 3962 (1972).

[44] P. G. Klemens, Anharmonic decay of optical phonon in diamond, Phys. Rev. B 11, 3206 (1975).

[45] S. Zhang, H. Zhang, T. Jia, Z. Wang, and Z. Sun, Coherent control of two-photon transitions in a two-level system with broadband absorption, Phys. Rev. A 80, 043402 (2009).

[46] J. H. Bechtel and W. L. Smith, Two-photon absorption in semiconductors with picosecond laser pulses, Phys. Rev. B 13, 3515 (1976).

[47] J. Oomens, B. G. Sartakov, G. Meijer, and G. von Helden, Gas-phase infrared multiple photon dissociation spectroscopy of mass-selected molecular ions, Int. J. Mass Spectrom. 254, 1 (2006).

[48] F. Eickemeyer, R. A. Kaindl, M. Woerner, T. Elsaesser, and A. M. Weiner, Controlled shaping of ultrafast electric field transients in the mid-infrared spectral range, Opt. Lett. 25, 1472 (2000).

[49] H. Katsuki, J. C. Delagnes, K. Hosaka, K. Ishioka, H. Chiba, E. S. Zijlstra, M. E. Garcia, H. Takahashi, K. Watanabe, M. Kitajima, Y. Matsumoto, K. G. Nakamura, and K. Ohmori, All-optical control and visualization of ultrafast twodimensional atomic motions in a single crystal of bismuth, Nat. Commun. 4, 2801 (2013). 